\title{
Do we still need CAVEs?
}

Guilherme Nunes de Vasconcelos ${ }^{1}$, Maria Lucia Malard ${ }^{2}$,

Mateus van Stralen ${ }^{3}$, Maurício Campomori ${ }^{4}$,

Sandro Canavezzi de Abreu ${ }^{5}$, Tales Lobosco ${ }^{6}$, Isabella Flach Gomes ${ }^{7}$,

Lucas Duarte Costa Lima ${ }^{8}$

1,2,3,4,5,6,7,8 Escola de Arquitetura, UFMG

1,3,5,8 \{guiazul|mateus-stralen|sandroid|lcostalima\}@ufmg.br

2,4,7\{mlmalard|mcampomori|isabellaflach\}@gmail.com6tales@lobosco.com.br

This paper discusses the relevance of CAVE systems in comparison with virtual and augmented reality head-mounted displays in terms of immersion experience, costs, maintenance, ease to use, interactivity, and social interaction. It is based on a comparative study of a systematic literature review comprising the works available at CumInCAD and IEEE databases in the period from 1998-2018, and empirical data from technical visits made to five CAVEs in Europe. The

discussion seeks to cover the limits of each technology and questions the need for CAVEs nowadays.

Keywords: CAVE, Virtual Reality, head mounted display, Augmented reality

\section{INTRODUCTION}

This article discusses the relevance of the Cave Automatic Virtual Environment (CAVE) systems in the context of the emerging opportunities brought by the 4th industrial revolution. The research is part of an ongoing development of a virtual reality (VR) training system for an electric power company in Brazil. The effectiveness of VR in education and training is being studied by authors from distinct fields (see Seymour, N. et. al. 2009; Henderson, S. et al. 2009; Pantelidis, V. 2009), however, the need for a CAVE has to be reevaluated in face of the recent development of more affordable VR technologies, such as the headmounted displays (HMD) for virtual and augmented realities. Thus, it seems necessary to ask if the rapid development of these devices will turn the CAVE unnecessary or does its strengths still justify its implementation and maintenance costs?

In this sense, this work proposes an investigation about the relevance of CAVE systems in the present days in comparison with other immersive technologies in terms of the immersion experience, costs, maintenance, ease of use, interactivity, and social interaction.

To support our analysis, we present a systematic literature review on CAVEs comprising 20 years of studies (1998-2018) from two important databases related to the field, CumingCAD and IEEE, and a report of our technical visits made to five CAVES in Europe. Afterward, we compare and discuss the literature review with our observations in order to offer our remarks about the relevance of CAVEs nowadays.

\section{A brief history of stereoscopic devices: from 1838 to nowadays}

In 1838, the English scientist and inventor, Sir Charles Wheatstone, published his paper "Contributions to Physiology of Vision" where he presented his discov- 
eries about the perception of three-dimensional objects. Observing how distance affects the perception of an object's depth, and how an object that is closer to an observer is seen differently by each eye, Wheatstone (1838) asked himself "What would be the visual effect of simultaneously presenting to each eye, instead of the object itself, its projection on a plane surface as it appears to that eye?" (Wheatstone, 1838).

To investigate this question he invented the stereoscope, an apparatus capable of presenting to an observer two distinct images of the same object to each observer's eye using an ingenious mechanism of mirrors and stands, tricking the mind to perceive a three-dimensional object from its bi-dimensional representations.

Wheatstone's experiment transformed our understanding of perception and stereoscopy is still the principle behind the most up-to-date technological immersive devices.

Thenceforth, a considerable number of stereoscopic devices were invented, of which the "Sword of Damocles" (Sutherland, 1968), is considered the first digital apparatus capable of tracking user's head position and rotation, and to present stereo images accordingly. Ever since, digital immersive devices became somehow popular through the work of many enthusiasts, such as Jaron Lanier (1999), who coined the term "virtual reality" from Sutherland's ideas presented in the paper "The Ultimate Display" (Sutherland, 1965), and Palmer Luckey, who developed the Oculus Rift, whose spark had revived interest in immersive technologies in the 21 st century (Purchese, 2013).

In 1992, a team of researchers led by Carolina Cruz-Neira, presented at the SIGGRAPH 1992 conference a room-based immersive technology intended for scientific visualizations, named CAVE, or Cave Automatic Virtual Environment (Cruz-Neira et al, 1992). The original CAVE consisted of a room with a set of projection screens for walls and floor, four projectors, workstations, speakers, a trackable stereo flicking glasses, and a trackable hand-controller, all synchronized with each other (Cruz-Neira et al, 1993). The capability of the CAVE to simulate environments and interact with digital information rapidly expanded its use from scientific visualization to training and education - mainly in fields that deal with dangerous conditions or involve high costs (Poschner, 2014). Among the alleged advantages of CAVEs are the multi-user possibility, a wider field of view, and the physical presence of users within the virtual environment (Manjrekar et al, 2014). The pointed disadvantages are mainly related to high implementation costs, maintenance, and space demands (Havig et al, 2011).

Since Cruz-Neira's first CAVE there have been several attempts to improve CAVE-based VR systems, including works such as CAVE 2.0, a hybrid (2D and 3D) virtual reality environment, that uses a circular installation with display walls consisting of a mosaic of high-resolution monitors (Febretti, 2014); TORE Antycip Simulation, the first ball room in the world, whose main features are in its screen shape, a half-sphere folded at the edges covering all dimensions to free users from any edges (Antycip Simulation, 2018); and the TENT, that differs from CAVE because, besides setting up a fluid, low cost, and ephemeral space, it creates a 3rd environment that interacts with the architecture of the place (Cabral Filho et al, 2006).

On the other direction, brought into focus by Luckey's Oculus Rift, many important technology companies have since 2015 announced the production of a wide range of portable immersive apparatuses, such as HTC Vive, Samsung GearVR, Sony Playstation VR Headset, Google Cardboard, and many others, in a phenomena that became known as "Virtual Reality (VR) third wave" (Heim, 2017). The prior waves occurred in the 1990s and at the turn of the 2000s (Heim, 2017).

This third wave of VR can be associated to the so-called "Industrie 4.0" (Drath, Horch, 2014), which refers to the "the triad of physical objects, their virtual representation and services and applications on top of those". The former industrial revolutions were respectively related to mechanization (the 1800s), electrification (the 1900s), and digitalization (the 1960s) 
(Schwab, 2006; Drath, Horch, 2014).

The developments in portable VR hardware were followed up by the evolution and popularization of game engines (software used to develop VR applications and, naturally, games), which became more accessible and user-friendly (Christopoulou et al, 2017). Presently, Unity and Unreal are the most widely used engines, with an active community and plenty of courseware available. This recent combination of more accessible hardware and user-friendly software contrasts with the previous implementation of VR solutions that needed custom hardware - and software.

Currently, a different form of immersive experience enabled by the recent development in augmented reality (AR) technology is gaining ground. What differentiates AR from VR is the possibility of presenting an interactive experience in a digital environment composed of digital and physical objects. Invented in the early 1990s, AR technology received significant updates in this last wave of development, becoming popular due to the possibility opened by smartphones to have AR experiences using its cameras and sensors. This technology became popular in 2016 with the mobile game Pokémon Go that allowed players to play with the game creatures while they were overlaid to the physical world in their smartphone's display. The game was downloaded more than 1 billion times since its launch (Webster, 2019). Nowadays, AR is incorporated in social network apps such as Instagram and Snapchat in the trendy "face filters", where it is possible to add digital props, enhance or transform one's face, creating somehow funny experiences.

Beside from smartphones, there are dedicated AR devices such as Microsoft Hololens, Magic Leap One, and Samsung Odyssey. These HMDs can present more immersive experiences, with increasingly display resolution and viewing angle. In comparison to VR HMDs, AR devices are less obtrusive since the user continues to see the physical world while interacts with physical objects and persons, as well as digital objects. Currently, the main technical challenge of AR HMDs lies in the relation between the size of the digital image and the field of view. However, devices such as Magic Leap One and Microsoft Hololens 2 are making great advances in this respect.

The developments in related immersive technologies apparently affected the CAVE devices comparatively little. The most significant developments in CAVEs are mainly related to incremental improvements in resolution, tracking systems, and graphics processing units. Different configurations of the CAVE were also developed, such as powerwalls (single plane interactive 3D display) and mobile CAVEs. Nevertheless, the main disadvantages attributed to CAVEs apparently have not been mitigated by those improvements.

This apparent mismatch between the technological developments of other immersion devices, such as the HMDs, and those related to CAVEs seems to point to a loss of interest or even the obsolescence of the latter. Thus, a systematic investigation through the literature about CAVEs can help to understand how it is perception and use has evolved over the years.

\section{METHODOLOGY}

The methodology combines literature review and qualitative data gathered in technical visits to five different CAVEs in three European countries. The review was mostly based in papers available at the CumInCAD and IEEE databases The CumInCAD gathers published papers from the main conferences relating Computer Aided Architectural Design, such as from ACADIA, CAADRIA, eCAADe, SIGraDi, ASCAAD, and $C A A D$, whilst IEEE is considered by many to be the world's largest organization for advanced technology.

Besides the chronological intent to cover the critical production of knowledge about the CAVEs, the information presented is aligned with the current thinking, as the literature review goes up to 2018.

The literature review is framed by a systematic examination of every article that contained the word CAVE, relating specifically to that device or to the development of some technology that would be tested 
using a CAVE, The papers were organized chronologically in order to analyze the relevance of the CAVE in studies from 1997 to 2018. Then, this review is compared with data from technical visits to different VR Labs (in industry and academia), software developers, and to five CAVEs located in France, the United Kingdom and Belgium. Those CAVEs were selected due to its proximity, availability for visits, diversity of configuration and uses, and different providers. The specific names and places of each CAVE will not be disclosed in this paper with respect to all those who have received us to show their products and visit their spaces. For this work, we understand that the report of our personal experiences and the descriptions of the technical characteristics of each CAVE were enough for the proposed discussion.

\section{Literature review: CumInCAD and IEEE CAVE related papers from 1998-2018}

In this review, we surveyed 145 papers from the last twenty years (1998-2018) available in the CumInCAD and IEEE databases, in which the CAVE appeared as a focus of research, whether in the field of education, experiment, training, innovation (in terms of CAVEs, software, and gadgets) and as a summary. However, for the current analysis, the focus of this work is on the papers that compare the characteristics of the CAVEs and the HMDs.

From CumlnCAD, we reviewed 41 (28,3\%) papers and $104(71,7 \%)$ from IEEE. The writings were organized chronologically and classified accordingly to their main focus as presented in its abstracts, as shown in Table 1: Innovation (new kinds of CAVE or gadgets), Education, Training, Representation (when the CAVE is used to exhibit some finding), Experiments with CAVEs, new Softwares, or Summaries of this medium.

On both databases the majority of the reviewed works, $60(41,4 \%)$ papers, were classified as Experiments, followed by Innovation with 29 (20\%) papers. In the sequence, $19(13,1 \%)$ works were classified as Education, 14 (9,6\%) papers as Training, Representation with $9(6,2 \%)$ papers, $8(5,6 \%)$ papers as Sum- mary, and finally $6(4,1 \%)$ works on Softwares, totalizing 145 (100\%) analyzed works.

\begin{tabular}{|c|c|c|c|}
\hline & CumInCAD & IEEE & TOTAL \\
\hline Experiments & $14(34,2 \%)$ & $46(44,2 \%)$ & $60(41.4 \%)$ \\
\hline Innovation & $7(17,2 \%)$ & $22(21,2 \%)$ & $29(20 \%)$ \\
\hline Education & $9(21,6 \%)$ & $10(9.6 \%)$ & $19(13,1 \%)$ \\
\hline Training & $1(2.5 \%)$ & $13(12,5 \%)$ & $14(9.6 \%)$ \\
\hline Representation & $8(19.5 \%)$ & $1(1,0 \%)$ & $9(6.2 \%)$ \\
\hline Summary & $1(2.5 \%)$ & $7(6.7 \%)$ & $8(5.6 \%)$ \\
\hline Softwares & $1(2.5 \%)$ & $5(4.8 \%)$ & $6(4.1 \%)$ \\
\hline TOTAL & $41(100 \%)$ & $104(100 \%)$ & $145(100 \%)$ \\
\hline
\end{tabular}

Figure 1

Classification of papers from CumInCAD and IEEE

Figure 2

Variation of categories during the period of 1998-2018
Figure 2 shows how the number of papers from each category varied in both databases during the period from 1998 to 2018 . Although a relatively steady increase in the number of papers of the categories of "Training" and "Summary", all other categories appears to decay following the first wave of interest, but they regain momentum in the last few years, especially "experiments", "innovation", "training" and "education", what could point to the impact of new technologies or the development of new applications or gadgets for this device.

With the intent to deepen the discussion, the most relevant papers that compare CAVEs with HMDs were analyzed. Ragan (2016) studied the effects of "Amplified Head Rotation" in VR and attested that the participants were able to maintain a better sense of 
spatial orientation using a CAVE, due to the visibility of the person's own body.

In agreement with the recent papers, Dalhom et al. (1999) identify the importance of the presence of the user's own body in the CAVE experience. For these authors, being physically present still promotes a deeper comprehension of attributes such as proximity, connectivity, and atmosphere. Göttig et al. (2004) also identify a bigger sense of presence and degree of isolation within a CAVE, compared to HMD, although they presented relatively similar spatial cognition and visual display quality impressions.

Borba (2017), in a work focused on the perception of advertisement in different immersive devices, showed that the feeling of presence was higher among the users of HMDs when compared to CAVEs, just as the feeling of being the avatar itself. Moreover, in accordance with Ragan (2016) and Dalhom et al. (1999), the sickness effect was only significant among HMD users. $\mathrm{Ng}$ (2017), studied how the lack of surrounding information in HMDs and the delay to process the next scenes can lead also lead to nausea.

Eloy et al. (2018) point out the importance of the available control mechanisms that could cause distractions and then disturbing the sense of presence. Moreover, the author found the CAVE more prone to reproduce the sense of presence, as it shows better results in the awareness of the controllers and display devices and the user adjustment to the virtual environment experience, although the powerwall presented less distracting controllers and the HMD presented more naturalistic mechanism for controlling the movement through the environment.

According to Schmidt (2018), a CAVE would offer a more comfortable experience and face-to-face communication. However, CAVEs does not allow the possibility to play against others, is not mobile, and it is considered expensive. Another disadvantage is that parts of the real world (such as the visible ceiling and ground) influences the sense of physical presence. This can explain why $91 \%$ of non-professional users that participated in this study have chosen the HMD in combination with the rowing simulator as their VR device of choice.

On the other hand, the study presented by $\mathrm{He}$ (2017) shows that HMD can be considered "over immersive" and it can distract the users, as well as the cables of HMDs can obstruct the motion.

It is interesting to notice that the comparison between CAVEs and HMDs is a current subject. The older papers had the goal to analyze the CAVE as a new technology and over the years, this device's advantages have been questioned by several authors, as cited. The literature review has shown that the main criteria used for comparison relate to immersion experience, such as interactivity, sense of presence, social interaction, etc. In the comparison section of this work, we return to these criteria and introduce others based on technical visits.

\section{Technical visits to CAVEs}

The visits described in this section are part of a bigger research project that aims to develop a gamified training platform for a state energy company (CEMIG) in Brazil. In this context, the building of two CAVEs (one at CEMIG and the other in our faculty) was initially proposed as the main interfaces to access the produced VR content. Accordingly, the technical visits here described were planned to provide researchers a first-hand experience with different CAVE configurations and understand if the high implementation and maintenance costs, as well as the other alleged disadvantages in technology, were justifiable in our case.

In total, five CAVEs were visited by the team. The first CAVE was located at a software development company and was intended for internal testing and showcasing their solutions. It was a low-cost CAVE with 3 projection planes ( 2 sides and floor) that used low-resolution ultra short throw projectors. Because it was intended for internal use, this CAVE was not well maintained and had problems with projection alignment in the projection surface corners. The lowresolution associated with the problems with the corners contributed to a low-grade immersive experience. 
The second CAVE, also located at a software development company, was a high-end demonstration CAVE with 4 projection planes (3 sides and floor). The CAVE was built with 4 "Christie's Mirage M" projectors, "ART" navigation system and "Volfoni 3D" glasses and emitters. In the demonstration, the CAVE was displaying a $3 \mathrm{~d}$ model of a car, as used in car-factories to review and visualize automobile designs. In this experience, it became clear how a welldesigned CAVE with high resolution and high contrast projectors is important for a good immersive experience. Nevertheless, it became also clear that the level of image distortion for people that are not using the tracked glasses can be quite confusing - even for a trained eye. Because the tracking systems only track one of the glasses, the rest of the viewers get a distorted perspective of what is being seen. In this visit, the hosts also showed a transportable powerwall, that fits a small briefcase and does not need any special knowledge to be ready to use.

The third CAVE, located at a University, is used for cutting edge interdisciplinary academic and industrial research, allowing teams of different origins and interests to study broad spectrum phenomena in digital scenarios, such as those potentially dangerous or expensive. It has the same configuration as the previous one, with 4 projection planes with Christie's projectors and the ART navigation system. The projection planes are made of large $10 \mathrm{~mm}$ acrylic plates that had to be put in place during the construction of the building because of their weight and size. The building was literally constructed around the CAVE.

Initially, this CAVE was run by a cluster of 4 computers running "Nvidia Quadro" GPUs. Later it was modified to work on a single CPU with an "AMD FirePro W9100" Graphics Processing Unit (GPU). This change was made to facilitate the use and maintenance of the CAVE by the technical employee. According to this employee, a good operational CAVE needs someone dedicated to constantly update and calibrate the system to enhance its use. Another feature of this CAVE was a 3D sound system. However, it was seldom used because of the difficulty to properly prepare the 3D sound for each different experiment.

Our experience in this CAVE was very positive, both because of the quality of the technical setup and the content. Different from the previous CAVE, where the content was focused on an object, we experienced two architectural spaces: a 3D representation of a medieval castle and an art gallery. From what we have experienced in this CAVE, the perception of immersion is much stronger when the focus is on space rather than when it relies on objects.

The fourth CAVE had the most unique technical setup because it was designed to be transportable. This transportable CAVE has 3 planes (two sides and floor) with hi-resolution Barco projectors and runs on a cluster of 4 computers ( 1 master and 3 slaves). Key components include projectors, projector brackets, mirrors, and screens. For each side of the CAVE, the main components are built into flight cases, which includes high-quality rear projection screen, projector, projector bracket, and mirror. The floor structure is stored in one side of suitcases, with the floor support structures located in the case on the other side. The company recommends storing the projectors, lenses, cables in their bags during transportation, for which another flight bag is provided. The two first flight cases measure around $3,0 \mathrm{~m} \times 1,0 \mathrm{~m} \times 2,5 \mathrm{~m}$ and the smaller one around 2,0m x 1,0m x 2,5m.

The transportable CAVE system is interesting if it is considered to be an almost off the shelf solution that can be assembled and disassembled with relative ease. According to the company technician, someone with experience can assemble the CAVE in one day of work, being necessary to calibrate the equipment, projectors, and screens at each assembly. However, given the dimensions of the equipment, rigid suitcases, the number of computers, added to the need to recurrently recalibrate the system, it is possible to conclude that the system is not configured as something truly easy to transport.

The fifth visit was to a state of the art CAVE mainly used to visualize architectural projects and details. The CAVE uses 25 Barco laser projectors to project overlapping images across five planes ( 3 
sides, floor, and ceiling). The system uses the Unreal Engine for running the content on 14 workstations with "NVIDIA Quadro" GPUs to update the scene in real time. Within the CAVE, a user controls the view with an ART joystick. The CAVE was designed in conjunction with the building and has a reception/meeting room with the control center, the CAVE environment, the technical environment with the projectors and a cooled basement for the computers.

This CAVE enabled the best immersive experience we had during the technical visits. This was greatly due to the quality and amplitude of the 5sided projections. The CAVE was big enough for several people to enter simultaneously which reinforced its social character. Nevertheless, also because of its size, the perspective distortions are quite big if you are not really close to the person using the head trackers.

When asked to compare CAVEs to HMDs the technicians explained that although HMDs have a good immersive quality they can be awkward for some people to use. According to them, some users can feel fragile by using HMDs because of the feeling that they are "cut from the real world".

\section{Complementary reports}

Other two companies and a laboratory working in VR related fields were also visited in the context of our research. One company works with technological development for VR, the other develop content for VR applications, and the laboratory is used for didactic purposes. In these visits, we also brought the discussion of the pertinence of a CAVE system for our specific case.

In addition to the aforementioned arguments of costs and maintenance, they pointed out that some companies are abandoning CAVEs in favor of other technologies. An example cited by one of the technicians is the Swedish National Road and Transport Research Institute (VTI) that had deactivated their CAVE and started using the same space with VR HMDs for their research due to its simplicity of use. Other mentioned example is the Dutch Faculty Fontys, that also closed their CAVE due to the low interest by students and companies, as most prefer to use HMDs because of its simplicity and cost difference. The researchers at Fontys are planning the development of a cheap, quickly buildable and to break down CAVE. However, there is no indication that it will happen soon.

\section{DISCUSSION}

The literature review has grounded the consideration of the CAVE as an instrument of research, education, and training. It was possible to attest that the matters of interest related to the CAVE have changed over the last 20 years. From the years 1998 to 2002 the most significant subject was Innovation and Education. The CAVE had been invented only six years before and therefore several researchers focused on the creation and adaptation of their own CAVE systems. Between 2003-2012 there was a stability of works; and in the last years, the interest in CAVE has increased in terms of matters as Experiments, Innovation (mainly related to new gadgets and Software), Training and Education. In the last few years, there has been an increase in papers that compare CAVEs and HMDs, attesting that there is clearly doubt about the use and implementation of CAVE systems.

Albeit CAVE technology has not been getting the same attention as VR and AR HMDs, it has received upgrades in order to improve technical aspects such as image resolution, projection and tracking systems technologies. Furthermore, there has been an effort to make CAVEs more accessible in cost, easier to maintain and in some cases make them transportable. However, it seems that many improvements were not directly driven by the CAVE industry. It is reasonable to suppose that many were driven by other actors, such as the cinema and game industry for example.

Nevertheless, even the improvements in transportability and ease of maintenance can be put into question. The transportable CAVE, for example, involves a somewhat complex operation to be installed, calibrated and transported (considering the weight and dimensions of the flight cases). Even 
though the transportable CAVE reveals an attempt to overcome certain constraints in technology and use when compared to the portability of HMDs, it still seems burdensome and unpractical.

The construction of a new CAVE is frequently a complex and costly task. Because of its footprint, two of the five CAVEs visited demanded planning that went back to the building design phase. The fact that in one of the visited CAVEs the structure and projection panels were transported to the building site in an early stage of construction so that only after that the walls of the CAVE room could be erected is symptomatic.

Besides that, the maintenance of a CAVE is laborious and demands specialized task, requiring, among other procedures, the alignment of projectors with the screen surface, the adjustment of projection corners - which is vital for immersion - and the synchronization of projections, operations that are recurrent and time-consuming. This is the reason that in every visited CAVE there was dedicated personnel to make the CAVEs working.

In comparison with HMDs, which are almost a "plug-n-play" solution, CAVEs are harder to set up, use, and maintain, demanding both physical and digital adjustment, as described above. Apart from the construction and equipment costs that are expensive, the dedicated personnel needed to keep CAVEs working adds an extra cost to this solution, making it even less viable than the HMDs.

In terms of immersion, our impressions from the technical visits are similar to what the literature review has pointed. Current HMDs devices offer a more immersive experience than CAVEs by blocking the visual perception of the physical world with a wideangle display placed close to the eyes. However, the distancing from the physical world can be distractive for some uses in situations such as collaborative work, training, and education.

Another aspect of immersion that differs HMDs in the comparison with CAVEs are the controllers used for interaction and the perception of the user's hands. In HMDs, when looking in the direction of the controllers, one sees the corresponding representation according to the content presented. The controllers can be viewed as a digital model of the controller itself, or a model of a hand, a model of a tool, or whatever the content creator imagined for that specific experience. In contrast, in CAVEs the user sees his own hand holding the controller overlapping a digital model that can vary in function of the content, which diminishes the sensation of immersion and sense of presence.

The CAVEs and HMDs controllers themselves present relevant differences. In recent years, the controllers for HMDs received improvements and features, such as finger-tracking, pressure, motion, touch, and optical data present in Valve Knuckles controller (Robertson, 2019). In contrast, CAVE controllers still rely mainly on motion tracking and buttons for interaction. Finally, Moehring et al. (2011) point out that CAVEs are more adequate for handling bigger objects, while HMDs are better for accurate and fine-grained manipulation.

The social interaction aspect enabled by CAVEs is, according to many authors, the greatest differential of the technology (Manjrekar et al, 2014; Schmidt, 2018, Mestre, 2017). The possibility of having a multiuser shared experience, face-to-face communication and interaction were used as an argument in favor of CAVEs by all technicians in our visits. However, we found that the shared experience can also be problematic, as in CAVEs the images are displayed according to a single user using the head-tracking. For the other users, the images appear distorted and sometimes incomprehensible. The single-user head tracking system used in CAVEs can also be problematic for face-to-face communication. Since the displayed images follow this user's head position, once he turns his head to talk with someone else, all the images are updated accordingly, interfering user's interaction with each other.

In HMDs the shared experiences are also possible, especially in multiplayer games, chat applications, and applications for collaborative working. However, the sickness effect and the sensation of iso- 
lation are still problematic in RV HMDs. This may change in the near future with AR HMDs that enabled a mixed experience of physical presence and the overlay of digital information, reducing sickness and isolation and amplifying the attributes of proximity, connectivity, and atmosphere.

\section{FINDINGS}

This work shows that although interest in immersive technologies has steadily increased in recent years, the development of high-quality VR and AR HMDs are pointing to a possible obsolescence of CAVEs system.

The improvements in VR and AR HMDs in controllers, display resolution, mobility, ease of use, costs, and maintenance, are making them preferable choices for companies and research centers.

The alleged advantages of CAVEs, such as social interaction and multi-user experience, were empirically questioned and do not seem to be the differential of this system anymore. The AR HMDs enable face-to-face communication in an almost nonobstructive way, as well as the possibility of sharing experiences with other users.

The use of new game engines, that are freely available and cloud-based, have enabled the creation of many VR applications. New features are constantly developed by a large online community which enabled the development of out of the box solutions. In contrast, many industries related to CAVE development did not follow this transition, relying on specialized knowledge, proprietary software, and expensive hardware.

Finally, the involved costs of equipment and personnel to implement a CAVE when compared to those related to HMDs, significantly reduce the chances to opt for a new CAVE. For this same reason, we believe that CAVE owners will not migrate to HMDs in the short term, in addition to the fact that CAVEs can still deliver immersive experiences that are suitable for some purposes.

In this sense, a question that still needs to be investigated in further research is related to who still needs CAVEs in the actual context where HMDs ap- pear as the obvious choice to make. Nonetheless, as far as we can see, the limits of the CAVEs are about to be reached while HMDs possibilities seem to be expanding.

\section{ACKNOWLEDGMENT}

This work was funded by ANEEL's R\&D Program through Cemig Distribution.

\section{REFERENCES}

Borba, EZ and Zuffo, MK 2017 'Advertising perception with immersive virtual reality devices', no source given, pp. 371-372

Christopoulou, E and Xinogalos, S 2017, 'Overview and Comparative Analysis of Game Engines for Desktop and Mobile Devices', International Journal of Serious Games, 4, p. 4

Cruz-Neira, C, Sandin, DJ and DeFanti, TA 1993 'Surround-screen projection-based virtual reality: the design and implementation of the CAVE', Proceedings of the 20th ACM Annual Conference on Computer Graphics and Interactive Techniques (SIGGRAPH) 93

Cruz-Neira, C, Sandin, DJ, DeFanti, TA, Kenyon, RV and Hart, JC 1992, 'The CAVE: audio visual experience automatic virtual environment', Communications of the ACM, 35(6), pp. 64-73

Dalholm, E, Rydberg-mitchell, B, Davies, R and Warrén, P 1999, 'The Experience of Space in Full-Scale Models and Virtual Reality', Proceedings 7th EFA-Conference., 7

Drath, R and Horch, A 2014, 'Industrie 4.0: Hit or Hype? [Industry Forum]', Industrial Electronics Magazine, IEEE, pp. 56-58

Eloy, S, Ourique, L, Woessner, U, Kieferle, J and Schotte, W 2018 'How present am I: three virtual reality facilities testing the fear of falling', eCAADe 2018, pp. 717-726

Febretti, A., Nishimoto, A., Thigpen, T., Talandis, J., Long, L and Sandin, D 2013 'CAVE2: a hybrid reality environment for immersive simulation and information analysis', The Engineering Reality of Virtual Reality 2013

Filho, C, J, BdS and P, A 2006, 'Tenda Digital/Digital TENT (Technological Environment for Negotiated Topology) e suas possíveis implicações em contextos sociais.', Proceedings of the 10th Iberoamerican Congress of Digital Graphics, 10, pp. 346-349 
Göttig, R, Newton, J and Kaufmann, S 2004, 'A Comparison of 3D Visualization Technologies and their User Interfaces with Data Specific to Architecture', in Van Leeuwen JP, TH (eds) 2004, Recent Advances in Design and Decision Support Systems in Architecture and Urban Planning, Springer, Dordrecht, pp. 99-111

Hamilton, I 2019, 'Facebook Bets On Realistic Face Tracked Avatars As Key To VR's Future', no title given

Havig, P, Mclntire, J and Geiselman, E 2011 'Virtual reality in a cave: limitations and the need for HMDs?', Headand Helmet-Mounted Displays XVI: Design and Applications

He, T, Chen, X, Chen, Z, Li, Y, Liu, S, Hou, J and He, Y 2017, 'Immersive and collaborative Taichi motion learning in various VR environments', InIEEE Virtual Reality (VR), pp. 307-308

Heim, MR 2017, 'Virtual Reality Wave 3', in surname missing, initials missing (eds) 2017, Boundaries of self and reality online: implications of digitally constructed realities, Academic Press, pp. 261-277

Henderson, SF 2009 'Evaluating the Benefits of Augmented Reality for Task Localization in Maintenance of an Armored Personnel Carrier Turret', 8th IEEE International Symposium on Mixed and Augmented Reality, pp. 135-144

Lanier, J 1999, 'Virtual Reality', Whole Earth Catalog,

Manjrekar, S, Sandilya, S, Bhosale, D, Kanchi, S, Pitkar, A and Gondhalekar, M( 2014 'An Emerging Immersive Technology-A Review', Computer Modelling and Simulation (UKSim), pp. 131-136

Mestre, DR 2017, 'CAVE versus Head-Mounted Displays: Ongoing thoughts', Electronic Imaging, The Engineering Reality of Virtual Reality, pp. 31-35

Moehring, M and Froehlich, B 2011, 'Natural interaction metaphors for functional validations of virtual car models', IEEE transactions on visualization and computer graphics, 17(9), pp. 1195-1208

$\mathrm{Ng}$, AK 2017 'Cognitive psychology and human factors engineering of virtual reality', 2017 IEEE Virtual Reality (VR), pp. 407-408

Pantelidis, V 2009, 'Reasons to Use Virtual Reality in Education and Training Courses and a Model to Determine When to Use Virtual Reality', Themes In Science And Technology Education, 2(1-2), pp. 59-70

Poschner, F 2014 'Fire fighting and related simulations in a CAVE using off-the-shelf hardware and software', Proceedings of SIGRADI 2014, Goteborg, Sweden, pp. 33-40

Purchese, R 2016, 'Happy Go Luckey: Meet the 20-yearold creator of Oculus Rift', EUROGAMER, 11
Ragan, E.D, Scerbo, S, Bacim, F and Bowman, D.A 2016 'Amplified head rotation in virtual reality and the effects on $3 d$ search, training transfer, and spatial orientation', IEEE transactions on visualization and computer graphics, pp. 1880-1895

Robertson, A 2019, 'The Valve Index might have the most fun VR controllers I've ever tried', The Verge

Schmidt, S, Ehrenbrink, P, Weiss, B, Voigt-Antons, JN, Kojic, T, Johnston, A and Möller, S 2018 'Impact of Virtual Environments on Motivation and Engagement During Exergames', Tenth International Conference on Quality of Multimedia Experience (QoMEX), pp. 1-6

Schwab, K 2006, 'The Fourth Industrial Revolution', World Economic Forum [online]. World Economic Forum

Seymour, NE, Gallagher, AG, Roman, SA, O'Brien, MK, Bansal, VK, Andersen, DK and Satava, RM 2002, 'Virtual reality training improves operating room performance: results of a randomized, double-blinded study', Annals of surgery, 236(4), pp. 458-464

Simulation, A 2018, 'Antycip Simulation Claims World-First in Virtual Reality for the University of Lille[Brochure]', no title given

Sutherland, I 1965 'The Ultimate Display', Proceedings of IFIP Congress, New York, pp. 506-508

Sutherland, I 1968 'A head mounted three dimensional display', Proceedings of the AFIPS Fall Joint Computer Conference, pp. 757-764

Webster, A 2019, 'no title given', Pokémon Go spurred an amazing era that continues with Sword and Shield

Wheatstone, C 1838, 'Contributions to the Physiology of Visio - Part the First On some remarkable, and hitherto unobserved Phenomena of Binocular Vision., in surname missing, initials missing (eds) 1838, no title given 